\title{
Direct Computation of a Hole-Tone Feedback System at Very Low Mach Numbers*
}

\author{
Kazuo MATSUURA** and Masami NAKANO*** \\ ** International Advanced Research and Education Organization, Tohoku University \\ 6-3 Aoba, Aramakiaza, Aoba-ku, Sendai, Miyagi, 980-8578, Japan \\ E-mail: matsuura@alba.ifs.tohoku.ac.jp \\ *** Institute of Fluid Science, Tohoku University \\ 2-1-1 Katahira, Aoba-ku, Sendai, Miyagi, 980-8577, Japan
}

\begin{abstract}
This study is concerned with an investigation into the sound produced when a jet, issued from a circular nozzle or hole in a plate, goes through a similar hole in a second plate. The sound, known as a hole-tone, is encountered in many practical engineering situation. Direct computations and experiments of a hole-tone feedback system are conducted. The mean velocities of the air-jet are 8 and $10 \mathrm{~m} / \mathrm{s}$ in the computations, and 6-13 m/s in the experiments. The diameters of the nozzle and the end plate hole are both $50 \mathrm{~mm}$, and the impingement length between the nozzle and the end plate is $50 \mathrm{~mm}$. The computational results agree well with the experimental data in terms of the qualitative vortical structures and the relationship between the most dominant hole-tone peak frequency and the jet speed. Based on the computational results of the air-jet speed of $10 \mathrm{~m} / \mathrm{s}$, the shear-layer impingement on the hole edge, the resultant propagation of pressure waves, the associated vortical structures and a newly proposed feedback mechanism is discussed. As far as the authors know this is the first direct computation of the hole-tone feedback system that predicts its dominant frequency successfully. Non-axisymmetry observed in the present computation is also shown.
\end{abstract}

Key words : Computational Fluid Dynamics, Hole Tone, Sound, Feedback Mechanism, Compressible Flows, Higher Order Scheme, Global Instability

\section{Introduction}

The sound produced when a jet, issued from a circular nozzle or hole in a plate, goes through a second plate with a hole of the same diameter as the jet is referred to as a hole-tone. We encounter the tone in many practical situations such as solid propellant rocket motors, automobile intakes and exhaust systems, ventilation systems, gas distribution systems, and whistling kettles, etc.

Sondhauss $^{(1)}$ first reported on the hole-tone in 1854 and found that the tone frequency increased with increasing jet velocity, and decreased with increasing distance between the orifices. Rayleigh $^{(2)}$, based on his observations, conjectured a mechanism, now known as a feedback mechanism ${ }^{(2-5)}$, that throws back the disturbances at the second plate, probably at the speed of sound, to the first plate where they give rise to further jet disturbances, which are then amplified by the unstable jet as they progress downstream.

In the mechanism, as a result of the axisymmetric instabilities of the jet each vortex is convected over the gap $L_{i m}$ during a time of the order of $L_{i m} / u_{c}$, at the shear-layer convection velocity $u_{c}$ that is typically about half the mean jet speed at exit $u_{0}$. An impulsive disturbance is generated when the vortex impinges on the downstream edge which initiates the formation of a new vortex. The impulse takes a finite time $\sim L_{\text {im }} / c_{0}$ to travel back across the gap, where $c_{0}$ is the speed of sound. Based on the picture the frequency $f$ of vortex formation satisfies $n / f \sim L_{i m} / u_{c}+L_{i m} / c_{0}$, where the values $n=1,2,3$, etc. correspond to the various stages

*Received 20 Jan., 2011 (No. 11-0063) [DOI: 10.1299/jfst.6.548]

Copyright (c) 2011 by JSME 
of operation $^{(3)}$. Howe $^{(6)}$ conducted linearized analyses of self-sustaining oscillations of high Reynolds number shear layers and jets incident on edges and corners at an infinitesimal Mach number. The operating stages of self-sustained oscillations are identified with poles in the upper half of the complex frequency plane of the Rayleigh conductivity. Umeda et al. ${ }^{(7)}$, in an experimental study on high subsonic jets, showed that although the feedback acoustic waves propagated upstream both inside and outside the jet, those outside were dominant. Langthjem and Nakano ${ }^{(8)}$ proposed a numerical approach to the hole-tone generation based on a discrete vortex method, using axisymmetric vortex rings combined with an acoustic feedback mechanism based on the Curl's equation.

Despite the long history of the hole-tone problem, a comprehensive analytical/numerical solution has not yet been developed. In this study, direct computations and experiments of a hole-tone feedback system at very low Mach numbers are conducted ${ }^{(9,10)}$. After validating the present numerical method against experimental data in terms of qualitative vortical structures and the relationship between the most dominant hole-tone peak frequency and the jet speed, this paper discusses the shear-layer impingement on the hole-edge, the resultant propagation of pressure waves, the associated vortical structures and a newly found feedback mechanism based on the computed results for an air-jet speed of $10 \mathrm{~m} / \mathrm{s}$. As far as the authors know this is the first direct computation of a hole-tone that predicts its dominant frequency successfully. In the hole-tone feedback system, the jet stability between the first and second plates is a key factor. In the previous papers ${ }^{(2,8,11)}$, instabilities of the jet have been considered to be axisymmetric. It is shown in this paper that non-axisymmetry is observed in the present computation when its results are precisely analyzed, as well as macroscopic feedback instability that is nearly axisymmetric.

\section{Numerical method}

The governing equations are the unsteady three-dimensional fully compressible NavierStokes equations in general coordinates. The perfect gas law closes the system of equations. Sutherland's formula for viscosity is adopted and a constant Prandtl number of $\mathrm{Pr}=0.72$ is assumed. The equations are solved using the finite-difference method. Spatial derivatives that appear in the metrics, convective and viscous terms are evaluated using the 6th-order tridiagonal compact scheme ${ }^{(12)}$. Near boundaries, the 4th-order one-sided and classical Padé scheme is used at the boundaries and one point internal to them. Time-accurate solutions to the governing equations were obtained using the 3rd-order explicit Runge-Kutta scheme. The time increment is constant in all flow fields. The CFL numbers of the present computations, which are defined by maximum sums of contravariant velocity and sound speed scaled by metrics, are around 0.4. In addition to the above-mentioned spatial discretization and time integration, a 10th-order implicit filtering ${ }^{(13)}$ is introduced to suppress numerical instabilities that arise from central differencing in the compact scheme. The filter parameters that appear in the left-hand side are set to be 0.33 for $i=2$ and imax $-1,0.492$ for $2<i<$ imax -1 . Near the boundaries, implicit filters of orders $p=(4,4,6,8,10)$ th for $i=(2, \cdots, 6)$ and $i=(\operatorname{imax}-1, \cdots, \operatorname{imax}-5)$, are used. Here, $i \in(k, l)$, and indices $j, k, l$ respectively run in the circumferential $\theta$, radial $r$ and streamwise $z$ directions in the general coordinate system. The present numerical method has been well validated for the prediction of transitional and turbulent subsonic flows ${ }^{(14)}$.

\section{Computational geometry and grid}

Figure 1 shows the computational overview of the present problem. The diameters of the nozzle and the end plate hole are both $d_{0}=50 \mathrm{~mm}$. In the numerical model the outer diameter of the end plate is taken to be $250 \mathrm{~mm}$. The reference gap length (or impingement length) $L_{i m}$ is $50 \mathrm{~mm}$. The reference mean velocities $u_{0}$ of the air-jet are 8 and $10 \mathrm{~m} / \mathrm{s}$. At $15^{\circ} \mathrm{C} u_{0}=10 \mathrm{~m} / \mathrm{s}$ corresponds to a Reynolds number $R e=u_{0} d_{0} / v=3.42 \times 10^{4}$ and a Mach number $M=u_{0} / c_{0}=0.029$, where the speed of sound $c_{0}=340 \mathrm{~m} / \mathrm{s}$ and the kinematic vis- 


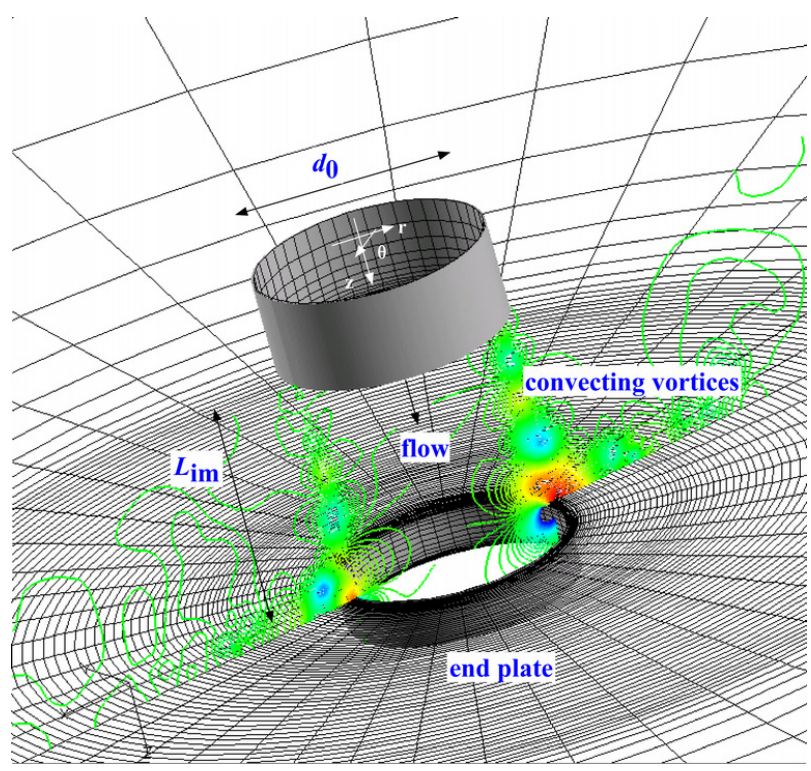

Fig. 1 Hole-tone system (An instantaneous pressure field in a computation when $\left.u_{0}=10 \mathrm{~m} / \mathrm{s}\right)$

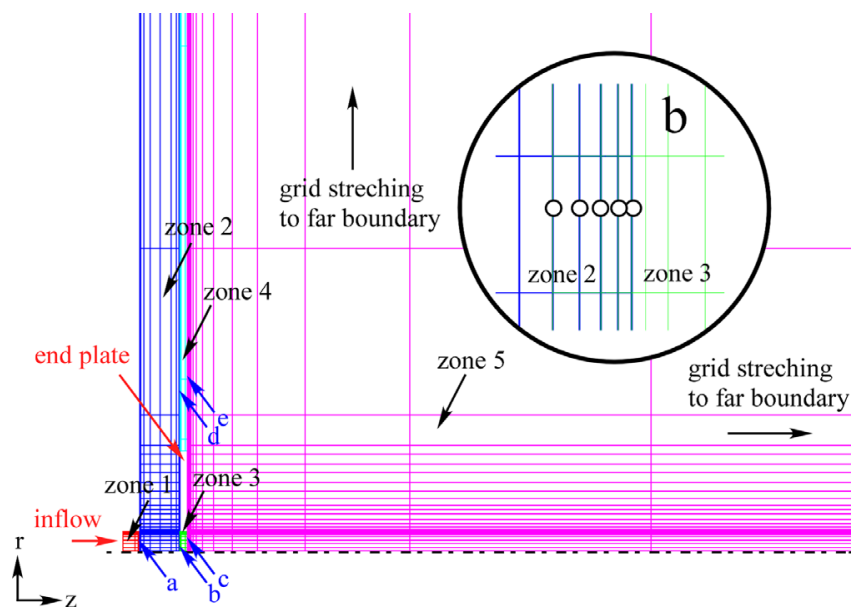

Fig. 2 Computational grid for $\theta=0$ rad (Every 5 grid lines is drawn for the $r$ and $z$ directions.)

$\operatorname{cosity} v=1.46 \times 10^{-5} \mathrm{~m}^{2} / \mathrm{s}$. Figure 2 shows the overview of the computational grid consisting of 5 zones, and cylindrical-coordinate grids $(r, \theta, z)$ of the O-type topology are generated in each zone. Exact overlapping of 5 grid lines in the normal direction to the interface, which corresponds to ' $a$, b , c, d, e' in Fig. 2, is used between neighboring zones. The details of overlapping for ' $b$ ' is shown in the enlarged figure. Periodicity in the $\theta$ direction is treated exactly without employing a one-sided biased scheme near the branch cut with regard to the derivative scheme. The present O-type topology has a singularity at the centerline. To circumvent this, primitive values circumferentially averaged at $k=2$ are inserted into primitive values at $k=1$, i.e, $f_{j, 1, l}=\frac{1}{\text { jmax }-1} \sum_{i=1}^{j \max -1} f_{i, 2, l}, \forall(j, l) \in(1, \cdots$, jmax $) \times(1, \cdots$, lmax $)$. The total number of grid points is basically $1.17 \times 10^{6}$ pts denoted as "grid A" and increased up to $9.07 \times 10^{6}$ pts denoted as "grid B" for checking grid independency of the results. In this study, the outer region of jets between the nozzle exit and the end plate is laminar or in an initial stage of transition when $u_{0}=8,10 \mathrm{~m} / \mathrm{s}$ as mentioned later. Therefore dominant/coherent structures under the present flow features with global instability are expected to be well predicted by a relatively small number of grid points if dispersion error is kept as minimum as possible. 


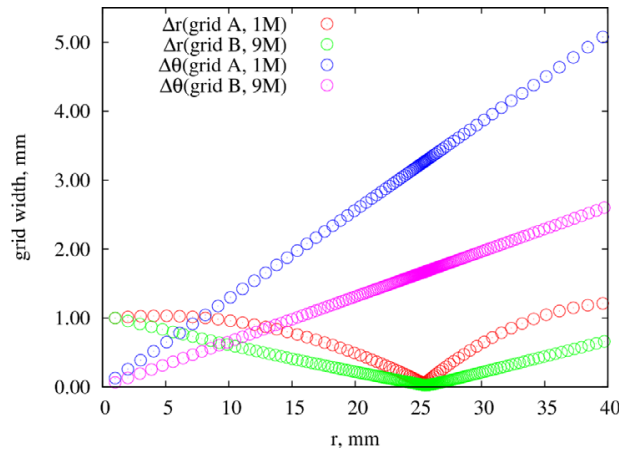

(a) $r, \theta$-dir.

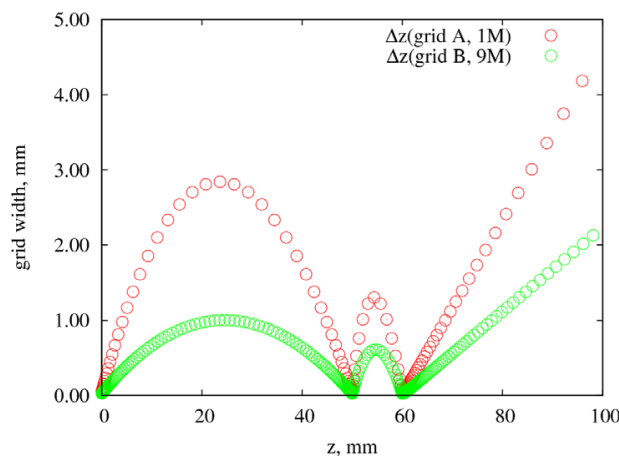

(b) $z$-dir.

Fig. 3 Distribution of grid width in the $r, \theta, z$ directions between the nozzle exit and end plate, inside the hole and downstream of the hole $(z=0 \mathrm{~mm}$ : nozzle exit)

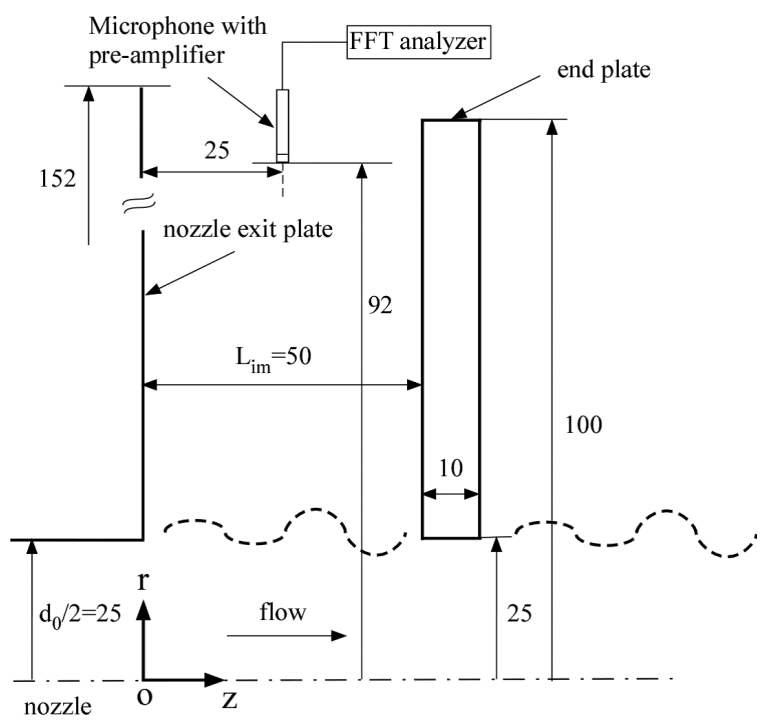

Fig. 4 Experimental system

Regarding the boundary conditions, inflow velocity profiles consistent with experimental data are specified at the nozzle inlet $\Omega_{z, \text { in }}=(r, \theta, z)=\left[d_{0} / 50, d_{0} / 2\right] \times[0,2 \pi) \times\left[-d_{0} / 2.5,-d_{0} / 2.5\right]$. Far boundaries are set at $\Omega_{z, e x}=\left[d_{0} / 50, d_{0} / 2\right] \times[0,2 \pi) \times\left[42.2 d_{0}, 42.2 d_{0}\right]$, and $\Omega_{r, e x}=$ $\left[42.5 d_{0}, 42.5 d_{0}\right] \times[0,2 \pi) \times\left[0,42.2 d_{0}\right]$. Here, $z=0 \mathrm{~mm}$ corresponds to the nozzle exit. At the far boundaries, grid stretching and non-reflecting conditions ${ }^{(15)}$ are used. The amplitude of pressure fluctuations near the far boundaries are attenuated to less than $O\left(10^{-4}\right)$ of the fluctuations near the hole. As an initial condition, a base profile and uniform random numbers $\epsilon_{i} \in[-1,1), \forall i \in(1, \cdots, 3)$ for breaking symmetry are employed.

$$
\left(\rho, u_{r}, u_{\theta}, u_{z}, p\right)=\left\{\begin{array}{l}
\left(\rho_{\infty}, 0,0, u_{0}, p_{\infty}\right)+\chi u_{0}\left(0, \epsilon_{1}, \epsilon_{2}, \epsilon_{3}, 0\right)|r|<d_{0} / 2 \\
\left(\rho_{\infty}, 0,0,0, p_{\infty}\right)|r| \geq d_{0} / 2
\end{array}\right.
$$

Here, $\chi$ is a scaling factor and set to be $1.0 \times 10^{-2}$. In the present system where global instability is taking place, shear layers near the nozzle exit are perturbed by pressure waves periodically propagating with natural oscillation frequency. To avoid disrupting naturally oscillating structures, no excitation such as a random forcing is imposed at the inlet boundary. The initial condition for grid B is prepared by interpolating the resultant flow fields of grid A. After the flow field reaches equilibrium, physical quantities are sampled for approximately $1.75 \times 10^{4} \mathrm{Lim}_{\text {im }} / \mathrm{c}_{0}$.

Figure 3 shows the distribution of grid points in both radial and streamwise directions between the nozzle exit and the end plate, inside the hole and downstream of the hole. Grid 
widths are made fine so as to capture the shear layer accurately in the $r$ direction, and separation region near both the nozzle exit and the end plate hole in the $\mathrm{z}$ direction. The maximum grid width $\Delta_{\max }$ near the jet is $\Delta_{\max } \sim 2.84 \mathrm{~mm}$ in the former grids as explained later in Fig. 3 (b). The minimum grid widths $\triangle_{\min } / d_{0}$ in these regions are $1 \times 10^{-3}$ in grid $\mathrm{A}$ and $4.9 \times 10^{-4}$ in grid B.

\section{Experimental apparatus}

The experimental system is shown in Fig. 4. The nozzle plate and the downstream end plate are rectangular, with dimensions of $300 \mathrm{~mm} \times 300 \mathrm{~mm}$ and $270 \mathrm{~mm} \times 200 \mathrm{~mm}$, respectively. Common to the computational geometry mentioned in Section 3, both the hole and end plate are acoustically compact. Air from a centrifugal blower goes through a $160 \mathrm{~mm}$ long mouthpiece before leaving the nozzle. When the end plate is removed, the ratio of the half width to the momentum thickness in the shear layers of the jet issuing from the nozzle exit are $R_{h} / \delta_{\theta}=6.44$ and 7.23 for $u_{0}=8$ and $10 \mathrm{~m} / \mathrm{s}$, respectively. Here, $R_{h}$ is the jet half width such that $u_{z}\left(R_{h}\right)=u_{0} / 2$, and $\delta_{\theta}$ is the momentum thickness. The shape factors of the boundary layer at the nozzle exit when the end plate is removed are 2.39, 2.33 and 2.33 for $u_{0}=8,10$ and $12 \mathrm{~m} / \mathrm{s}$, respectively, and the boundary layer states at the nozzle exit are laminar for all the jet velocities. The intensity of the free-stream disturbance at the nozzle exit when the end plate is removed is approximately $1.3-1.7 \%$ for the above jet velocities.

Sound pressure levels are measured by a condenser microphone. Data are passed through a microphone preamplifier to a FFT analyzer. To take instantaneous snapshots of the jet shear-layers, a laser sheet is used with a high-speed video camera by injecting the smoke of evaporated glycol solvent into the jets. The thickness of the laser sheet is approximately 1 $\mathrm{mm}$. It has been established that the sheet goes through the center of the hole. The sampling rate of the snapshots is 1500 frames/s. An I-type hotwire is also used to measure velocity fluctuations in the jet shear layers. It has been established that the insertion of the hotwire does not influence the hole-tone frequency.

\section{Results and discussion}

\subsection{Macroscopic feedback mechanism and subharmonic modulation}

Figure 5 shows experimental time variations of jet behaviors every $T_{f} / 4$ between the nozzle exit and the end plate for $u_{0}=4-10 \mathrm{~m} / \mathrm{s}$, and successive snapshots at $1500 \mathrm{fps}$ for $u_{0}=12 \mathrm{~m} / \mathrm{s}$. Here, $T_{f}$ is the period of the most dominant hole-tone peak frequency for each jet velocity. Figure 6 shows the measured power spectrum density function of $\sqrt{u_{r}^{2}+u_{z}^{2}}$ at $(r, z)=\left(d_{0} / 2+1, L_{i m} / 2\right)$. When $u_{0}=4 \mathrm{~m} / \mathrm{s}$, sinuous motion of the jet is observed in Fig. 5 . When the jet speed is increased, rolling up of the shear-layer via Kelvin-Helmholtz instability occurs, and the shear-layer impinges on the edge of the end plate hole. Non-axisymmetric vortices, i.e., helical disturbances near in jet shear layer, are also observed. As the jet speed is increased, the number of vortices increases. Until $u_{0}=10 \mathrm{~m} / \mathrm{s}$ the shear layer is laminar based on Figs. 6(a) and (b), and the shape factors mentioned in Section 4. When $u_{0}=12 \mathrm{~m} / \mathrm{s}$ the shear layer becomes intermittently turbulent as found from broad band fluctuations in 6(c), and a mode jump in the dominant peak frequency of the hole-tone takes place, which is explained later in Fig. 7. When these experimental flow behaviors are compared with the computational results, flow features such as rolling up of the shear-layer, shear-layer impingement on the edge of the end plate hole, and non-axisymmetric vortices are successfully reproduced in the present computation as shown in Fig. 1, and later in Figs. 12 and 13.

Figure 7 shows the variation of the most dominant hole-tone peak frequency with the jet speed. The peak frequencies increase nearly in proportional to $u_{0}$, and therefore, self-sustained oscillation without acoustic resonance with the wind tunnel is taking place. In the figure, the Rossiter's equation $n / f=L_{i m} / u_{c}+L_{i m} / c_{0}$ mentioned in Section 1 is also plotted assuming $u_{c}=0.6 c_{0}$ in addition to the experimental and computational data. When $u_{0}$ is increased monotonically from $6 \mathrm{~m} / \mathrm{s}$, a mode jump takes place around $u_{0}=12 \mathrm{~m} / \mathrm{s}$. Conversely, when $u_{0}$ 

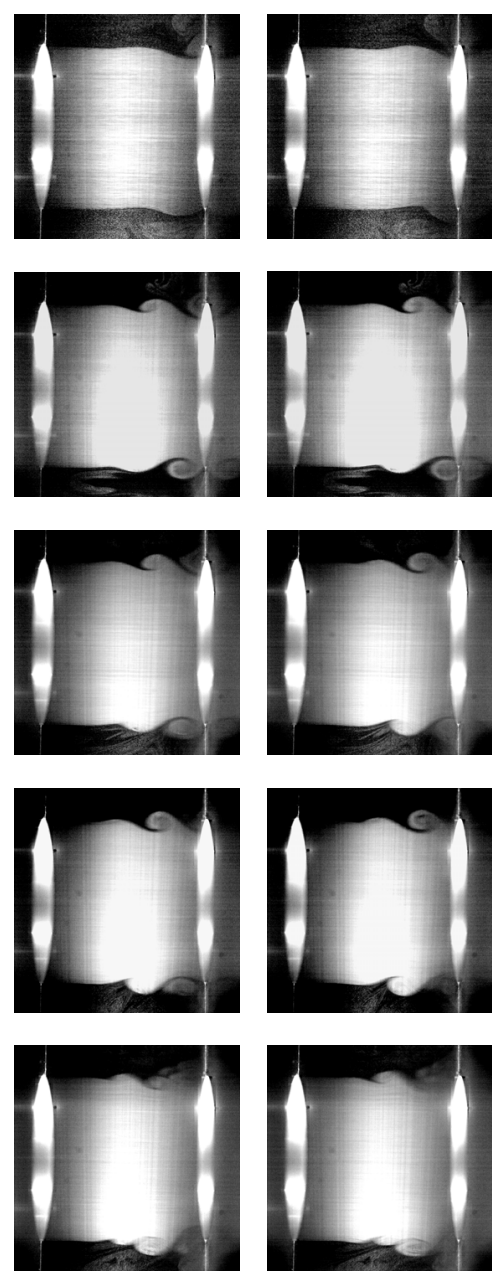

Fig. 5
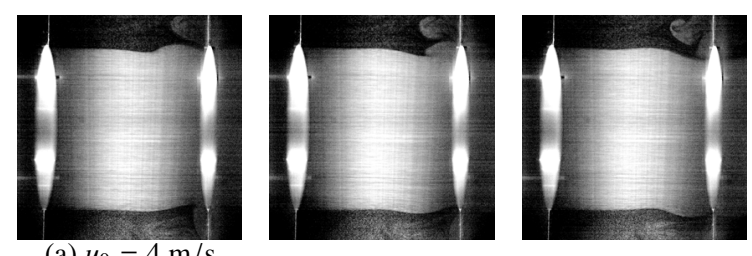

(a) $u_{0}=4 \mathrm{~m} / \mathrm{s}$
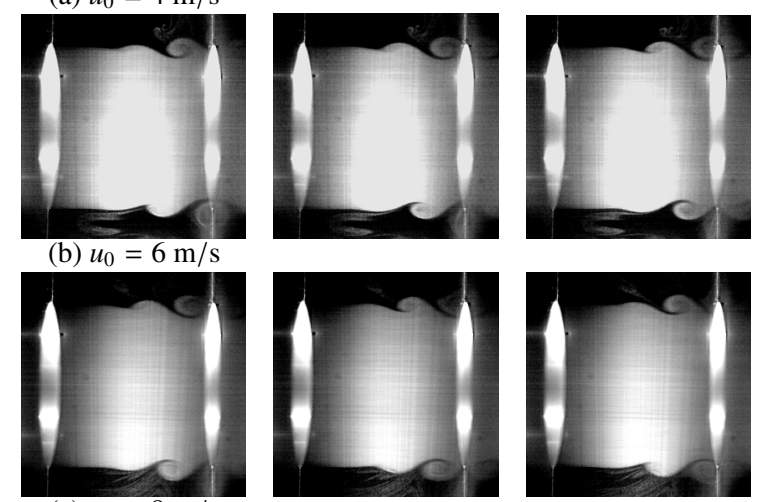

(c) $u_{0}=8 \mathrm{~m} / \mathrm{s}$
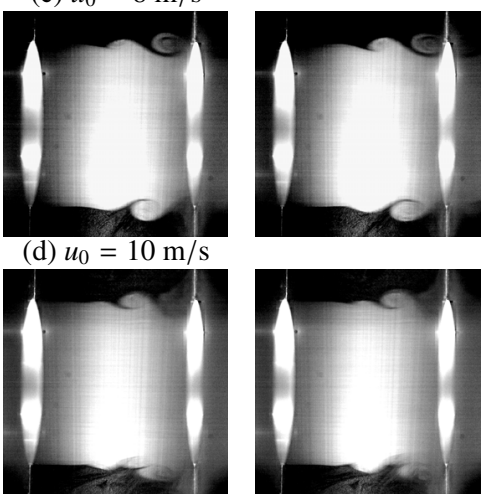

(e) $u_{0}=12 \mathrm{~m} / \mathrm{s}$
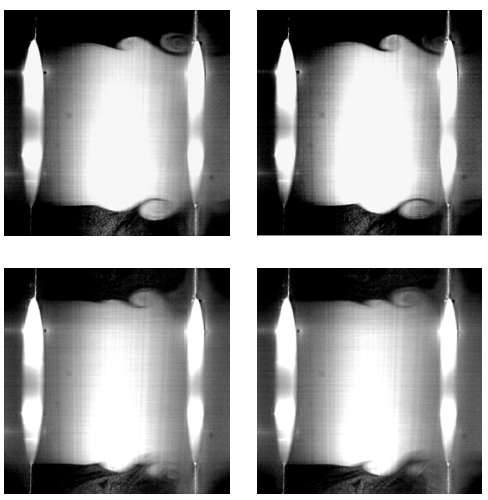

Experimental visualization of jet between the nozzle exit and the end plate for $u_{0}=4-12 \mathrm{~m} / \mathrm{s}, t=0$ (most left), $T_{f} / 4,2 T_{f} / 4,3 T_{f} / 4, T_{f}$ (most right) for $u_{0}=4-10 \mathrm{~m} / \mathrm{s}$, and successive snapshots at $1500 \mathrm{fps}$ for $u_{0}=12 \mathrm{~m} / \mathrm{s}$

is monotonically decreased from $12 \mathrm{~m} / \mathrm{s}$, a mode jump takes place around $u_{0}=10 \mathrm{~m} / \mathrm{s}$. Thus, the experimental results show hysteresis around $u_{0}=10-12 \mathrm{~m} / \mathrm{s}$. The present computations with relatively coarse grid A accurately predict the peak frequency of $u_{0}=8 \mathrm{~m} / \mathrm{s}$, and that of $u_{0}=10 \mathrm{~m} / \mathrm{s}$ when the jet speed is increased. Computations with finer grid B gives the same peak frequency. So, the results obtained at $u_{0}=10 \mathrm{~m} / \mathrm{s}$ with grid A are discussed hereafter.

Figure 8 shows the time variation of the pressure fluctuation $\Delta p$ distribution upstream and downstream the end plate where $\Delta p=p-p_{\infty}$ and $p_{\infty}=101,325 \mathrm{~Pa}$. The region outside the range $-3 \leq \Delta p \leq 3$ is excluded in order to emphasize the relative background pressure distribution between the space upstream of the end plate and the space downstream of it. High and low pressure regions alternately appear over a wide area upstream and downstream of the end plate, and the hole part becomes a node for the change. Pressure waves pass through the jet, and the jet including its shear-layers oscillates periodically. Figure 9 shows space-time plots, i.e., the time evolution of the pressure fluctuation distribution at $r=5,20,40,94 \mathrm{~mm}$. Here, $r=5 \mathrm{~mm}$ corresponds to the neighborhood of the centerline, $r=20 \mathrm{~mm}$ corresponds to the jet shear layer, and $r=40$ and $94 \mathrm{~mm}$ corresponds outside of the hole. The ordinate is time $t^{*}$ non-dimensionalized by $L_{i m} / c_{0}$ and the abscissa is the $\mathrm{z}$ coordinate, where $z=0 \mathrm{~mm}$ corresponds to the nozzle exit as explained in Fig. 4. The times $t=0, T_{f}, 2 T_{f}$ in Fig. 8 correspond to the times $t^{*}=0,1191,2396$ respectively in Fig. 9. The sound speed propagation from downstream to upstream is shown by the arrow with $c_{0}$. At $r=5 \mathrm{~mm}$, a high-pressure 


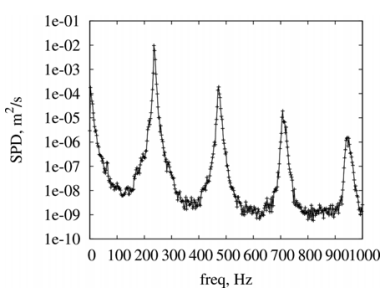

(a) $u_{0}=8 \mathrm{~m} / \mathrm{s}$.

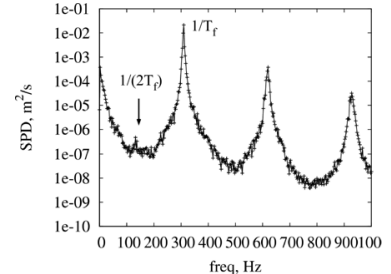

(b) $u_{0}=10 \mathrm{~m} / \mathrm{s}$.

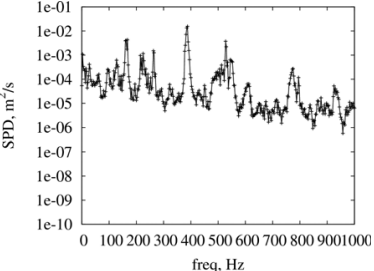

(c) $u_{0}=12 \mathrm{~m} / \mathrm{s}$.

Fig. 6 Measured power spectrum density function of $\sqrt{u_{r}^{2}+u_{z}^{2}}$ at $(r, z)=\left(d_{0} / 2+\right.$ $\left.1, L_{i m} / 2\right)$

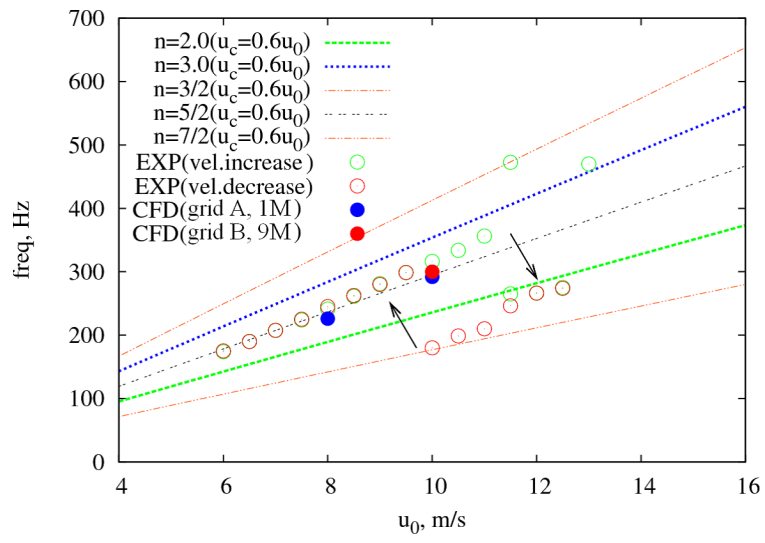

Fig. 7 Variation of the most dominant hole-tone peak frequency with jet speed $u_{0}$

region " $\mathrm{A}$ " is formed inside and downstream from the end plate hole, and a low-pressure region " $\mathrm{B}$ " is then formed near the nozzle exit and propagates downstream as found by the inclination of the region. Then a high-pressure region " $\mathrm{C}$ " is formed just before the end plate hole, and propagates upstream. When Fig. 9 is compared with Fig. 8, the regions "A" and "B" approximately corresponds to the pressure distribution of Fig. 8(e). From Figs. 8(f),(g), the appearance of region " $\mathrm{C}$ " is caused by the appearance of the high pressure region shown as "high" in Figs. 8(f) and (g) near the hole edge. At $r=20 \mathrm{~mm}$, high and low pressure regions convect downstream slowly with the convection velocity of approximately $0.69 u_{0}$. At $r=40$ and $94 \mathrm{~mm}$, pressure waves become almost planar. Figure 10 shows a power spectrum density function of the pressure fluctuation $\Delta p$ at $r=d_{0} / 2, \theta=0 \mathrm{rad}$ and $z=L_{\text {im }} / 2$. In the figure, in addition to the fundamental frequency $f_{0}$, the subharmonic frequency $f_{0} / 2$, which is considered to be vortex paring and consistent to Fig. 6(b), is also observed. With Figs. 8, 9 and 10 both taken into account, dominant pressure fluctuations in the present feedback system propagate inside the jet with alternate appearance of wide high and low pressure regions before and after the end plate hole, and the frequencies of the pressure fluctuations and vortices in the shear layer coincide. It has been believed for some time that pressure waves outside the jet trigger the feedback of the hole-tone regarding the feedback mechanism mentioned in Section 1. The global phenomena explained above are newly discovered feedback phenomena.

Figures 11 and 12 show the variations of pressure and velocity fields due to shear-layer impingement on the hole edge for $t=0-2 T_{f}$, and vortical structures visualized by the isosurfaces of the second invariance of the velocity gradient tensor $Q^{*}=29.0$, respectively. Here, $Q^{*}$ is defined as $Q^{*}=\frac{1}{2}\left(-S_{i j}^{*} S_{j i}^{*}+\Omega_{i j}^{*} \Omega_{i j}^{*}\right)$, where $S_{i j}^{*}$ is the rate of strain tensor and $\Omega_{i j}^{*}$ is the vorticity tensor. $Q^{*}, S_{i j}^{*}$, $\Omega_{i j}^{*}$ are non-dimensionalized by $u_{0}$ and $L_{i m}$. The time $t$ in Figs. 11 and 12 is equal to $t$ in Figs. 8 and 10. In Fig. 11 high and low pressure regions alternately approach the hole edge, and very high pressure regions denoted as " $\mathrm{D}$ " are generated around $t=0, T_{f}$ and $2 T_{f}$. As the high pressure region near the hole edge diminishes, a low pressure region denoted as "G" separates from the edge, and eventually the region leaves the hole as 


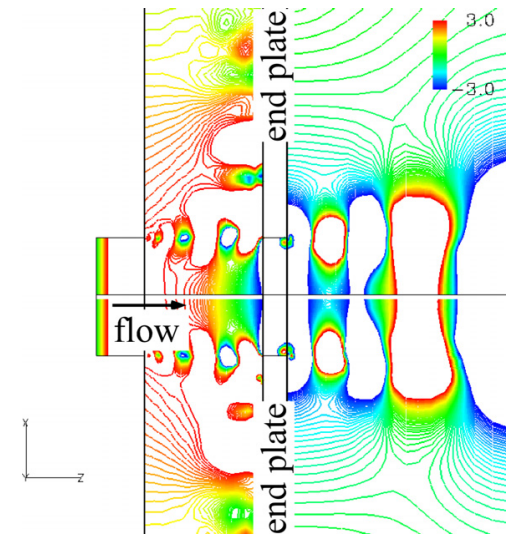

(a) $t=0$

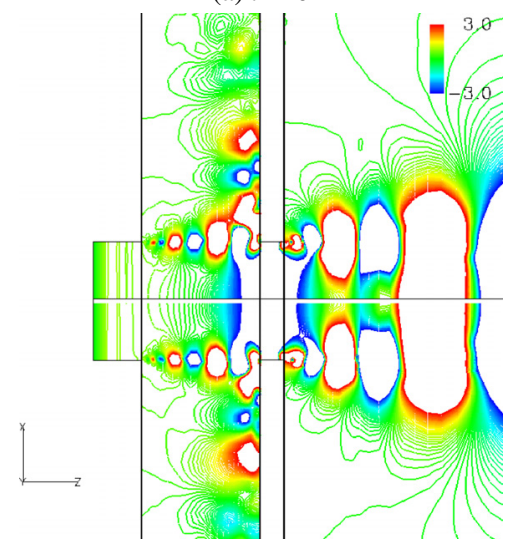

(c) $t=2 T_{f} / 6$

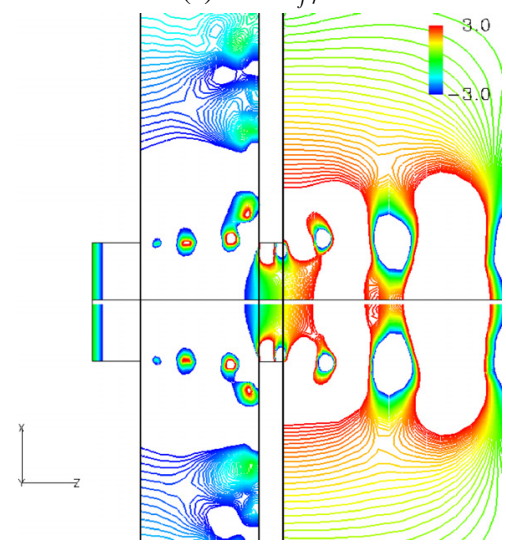

(e) $t=4 T_{f} / 6$

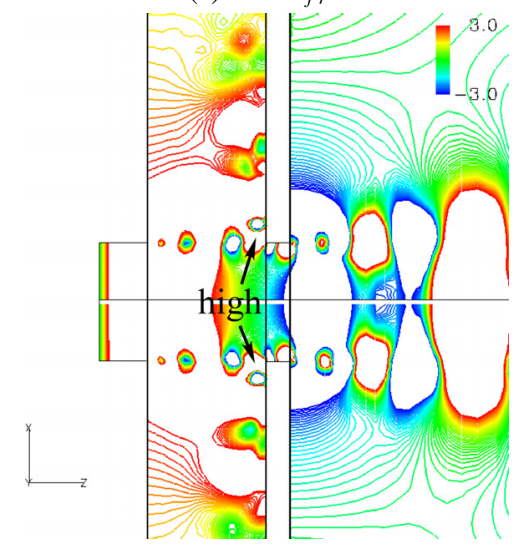

(g) $t=6 T_{f} / 6$

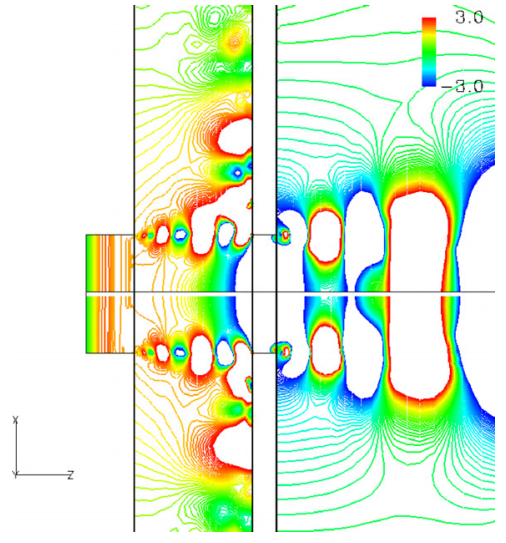

(b) $t=T_{f} / 6$

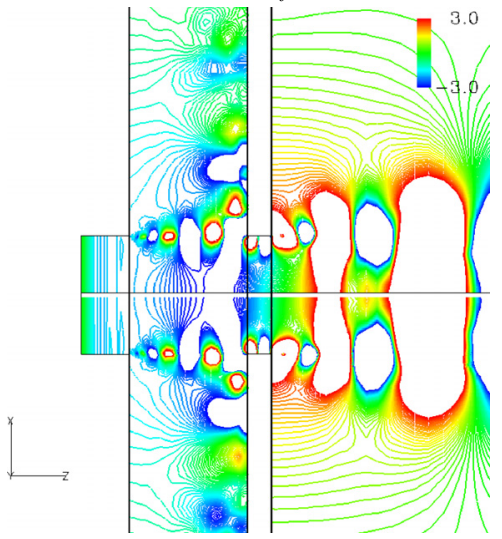

(d) $t=3 T_{f} / 6$

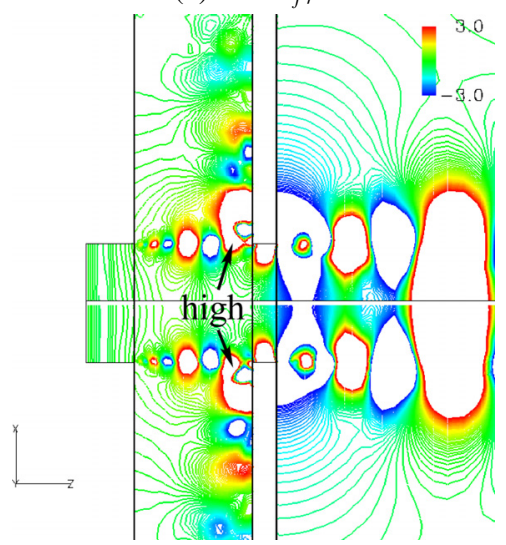

(f) $t=5 T_{f} / 6$

Fig. 8 Time variation of pressure fluctuation $\Delta p=p-p_{\infty}, p_{\infty}=101,325 \mathrm{~Pa}$ distribution upstream and downstream the end plate when $u_{0}=10 \mathrm{~m} / \mathrm{s}\left(1 / T_{f} \sim\right.$ $292 \mathrm{~Hz})$ 


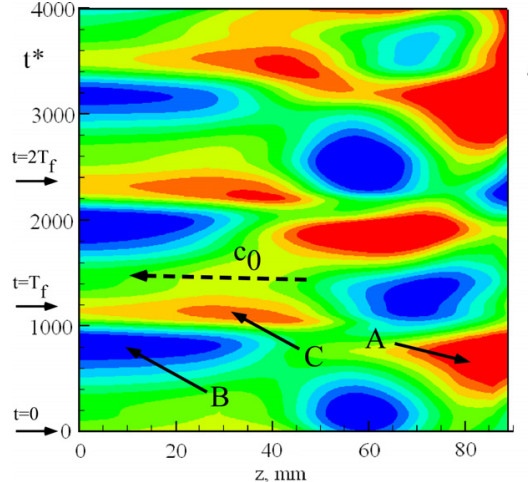

(a) $r=5 \mathrm{~mm}$

(near centerline)

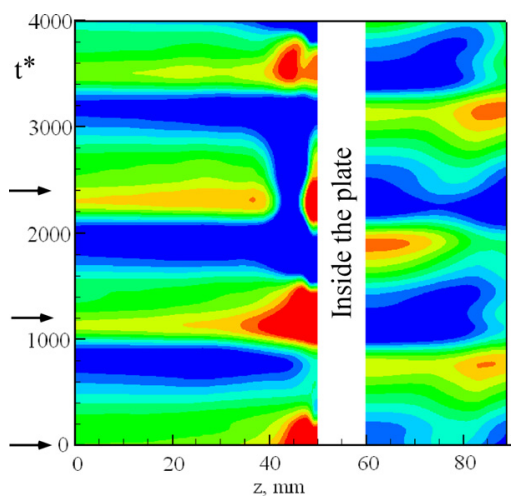

(c) $r=40 \mathrm{~mm}$

(outside the hole; closer to the centerline)

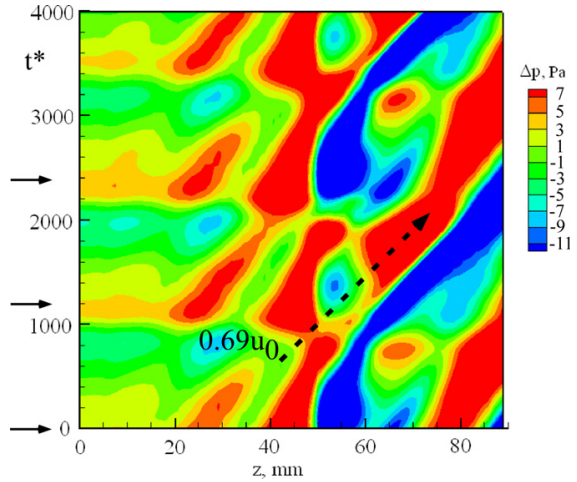

(b) $r=20 \mathrm{~mm}$

(near jet shear-layer)

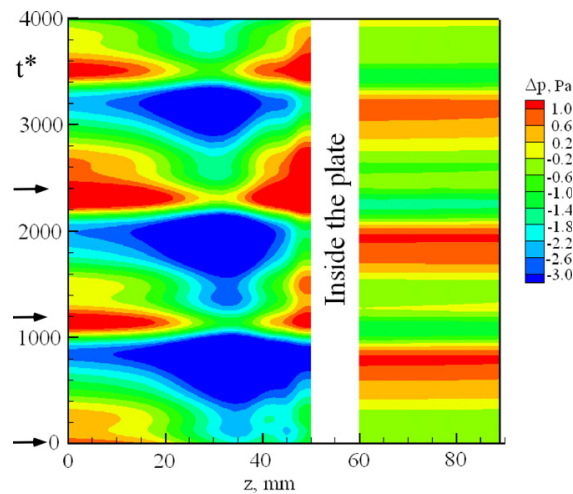

(d) $r=94 \mathrm{~mm}$

(outside the hole; far from the centerline)

Fig. 9 Space-time plots of pressure fluctuation $\Delta p$ at $r=5,20,40,94 \mathrm{~mm}(z=0$ : nozzle exit)

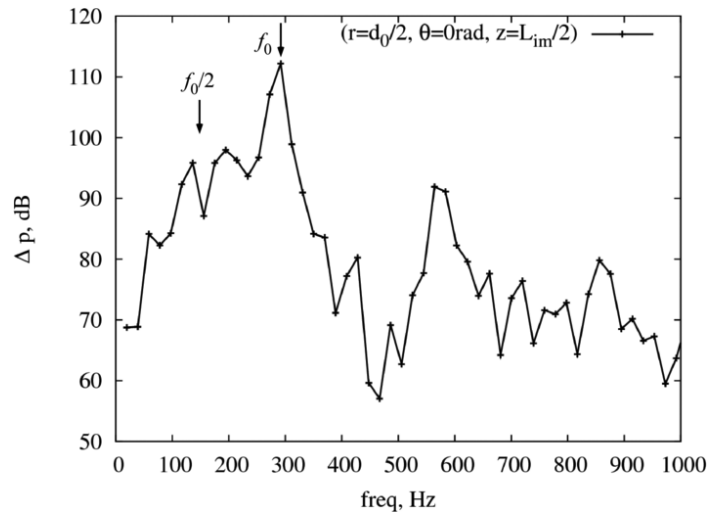

Fig. 10 Power spectrum density function of pressure fluctuation $\Delta p$ at $(r, z)=$ $\left(d_{0} / 2, L_{\text {im }} / 2\right)$ 


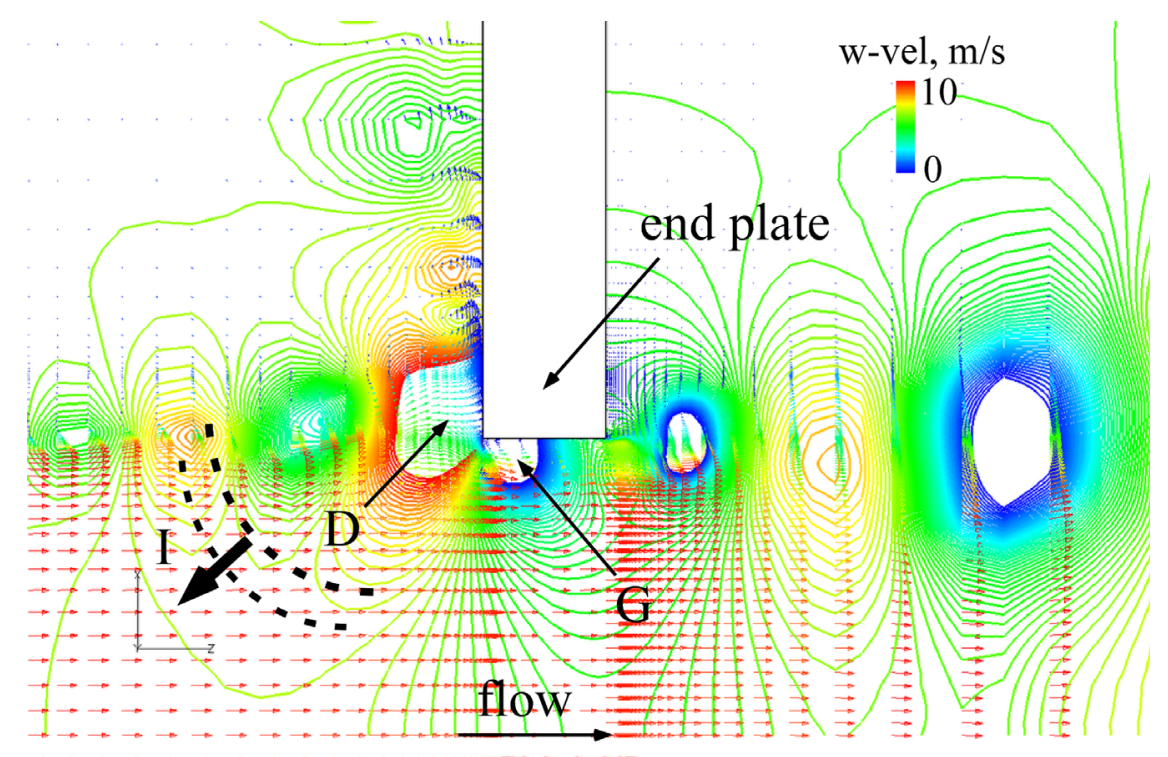

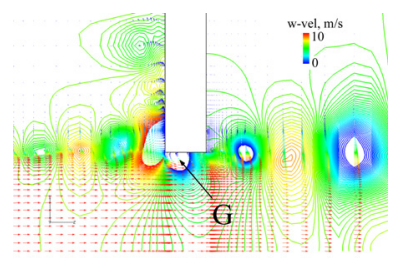

(b) $t=T_{f} / 6$

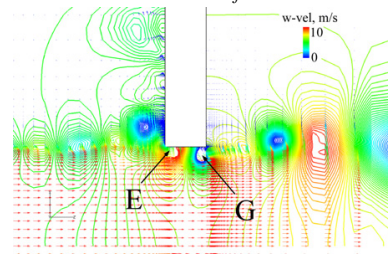

(e) $t=4 T_{f} / 6$

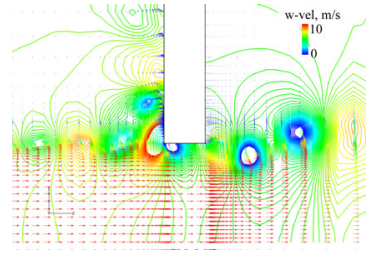

(h) $t=7 T_{f} / 6$

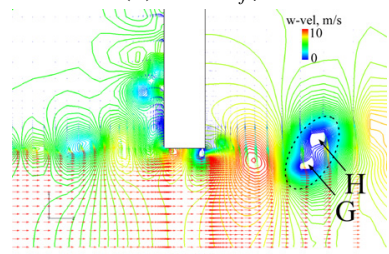

(k) $t=10 T_{f} / 6$

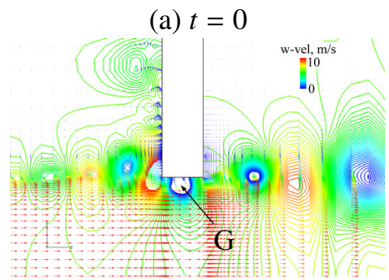

(c) $t=2 T_{f} / 6$

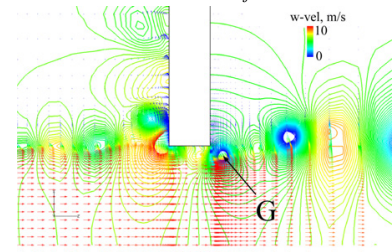

(f) $t=5 T_{f} / 6$

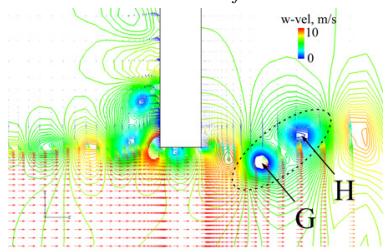

(i) $t=8 T_{f} / 6$

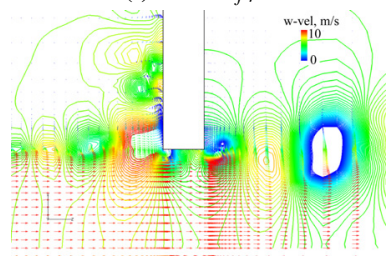

(1) $t=11 T_{f} / 6$

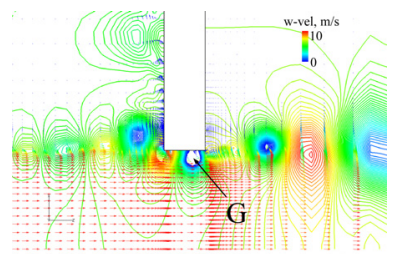

(d) $t=3 T_{f} / 6$

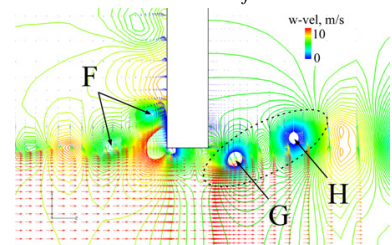

(g) $t=6 T_{f} / 6$

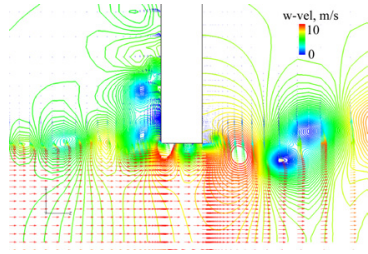

(j) $t=9 T_{f} / 6$

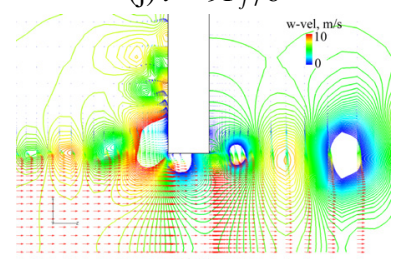

(m) $t=12 T_{f} / 6$

Fig. 11 Time variation of pressure and velocity fields due to shear-layer impingement on the hole edge $\left(1 / T_{f} \sim 292 \mathrm{~Hz}, \Delta p=-30\right.$ (blue) -30 (red) Pa) 
found in Figs. 11(a)-(f).

In summarizing Figs. 8-12, the feedback process in the present computation is interpreted as follows. (1) At $t=0$, the high pressure region ("D" in Fig. 11(a)) appears between the centers of successive vortices near the upstream side of the hole edge due to the vortex impingement. Then, the induced pressure waves propagate upstream inside the jet ("C" in Fig. 9(a), "I" in Fig. 11(a)). As a result, the background pressure in the space between the nozzle exit plate and the end plate increases. While, the background pressure downstream of the end plate hole becomes almost minimum (Fig. 8(a)). (2) For $t \sim 1 T_{f} / 6-2 T_{f} / 6$, because the high background pressure between the nozzle exit plate and the end plate pushes the jet flow, the large separation denoted by "G" in Figs. 11(b) and (c) takes place inside the end plate hole, and the background pressure in the space between the nozzle exit plate and the end plate starts to decrease (Figs. 8(b) and (c)). (3) Because the jet flow is pushed outside through the end plate hole, high pressure regions gradually appear near the exit of the hole (Figs. 8(c) and (d)). At $t \sim 4 T_{f} / 6$ (Fig. 8(e)), the background pressure between the nozzle exit plate and the end plate becomes almost minimum, while the background pressure outside of the end plate hole becomes almost maximum. (4) The high pressure regions denoted by "high" in Fig. 8(f) begin to grow again near the upstream side of the hole edge due to the vortex impingement, and the background pressure in the space between the nozzle exit plate and the end plate starts to increase. (5) The above mentioned fluctuation of the background pressure near the nozzle exit affects the jet shear-layer disturbances there. (6) The shear-layer disturbances convect downstream and grow to form vortices. (7) Process (1) is again repeated.

The present feedback phenomena consists of four elements, i.e., birth of disturbances in the jet shear layer, convection and amplification of disturbances leading to form vortices, generation of pressure waves due to the vortex impingement, upstream propagation of the pressure waves inside the jet for the birth of disturbances. It might be important to maintain the feedback loop that the vortex impingement, associated with the alternative global fluctuations of the background pressures between the upstream and downstream sides of the end plate, generates the periodic fluctuation of pressure waves near the upstream edge of the endplate hole, which are propagating upstream inside of the jet. While the former two elements observed in the present paper does not change past views ${ }^{(2,4)}$, new aspects of the mutual relationship between the latter two elements are given in the above feedback process (1)-(7).

In the paper of Langthjem and Nakano ${ }^{(8)}$, acoustic feedback is modelled by adding acoustic perturbation velocities to velocities near the nozzle exit, and the acoustic perturbation velocities are evaluated by a simplified Curl's equation where only dipoles and monopoles are retained. The dipoles and monopoles are evaluated respectively by the fluid force acting on the upstream surface of the end plate, and by the time derivative of velocity at the hole inlet position. Compared to the feedback model, the newly found feedback process in the present study involves wide area over the upstream and downstream of the end plate hole.

While the dominant frequency of the macroscopic feedback cycle is $f_{0}$, subharmonic modulation is also observed in the present study as mentioned previously in Fig. 10. In Figs. 11(a)-(m), the subharmonic modulation corresponds to the accumulation of vortices " $F$ " in Fig. 11(g) and the resultant pairing of vortices " $\mathrm{G}$ " and " $\mathrm{H}$ " in Figs. 11(g)-(k) and Figs. 12(a)-(f).

\subsection{Non-axisymmetric instability}

Finally, a non-axisymmetry observed in the present computation is noted. Figure 13 shows the polar plots of $\Delta p$ at $r=d_{0} / 2, z=L_{i m} / 2$ for the times when the pressure fluctuation becomes a local maximum. In the figure, there are deflections of no more than $5 \mathrm{~Pa}$ during $t^{*}=990-9344$. In Fig. 12 slight tilting of the vortex rings are observed both upstream and downstream of the end plate. While axisymmetric instabilities have been exclusively reported in hole-tone studies, there are possibilities for non-axisymmetric disturbances as its global instability. 


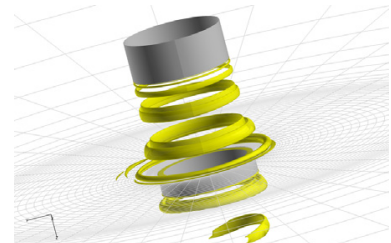

(a) $t=0$

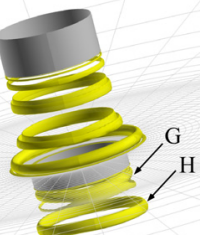

(d) $t=6 T_{f} / 6$

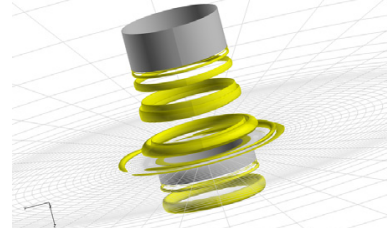

(b) $t=2 T_{f} / 6$

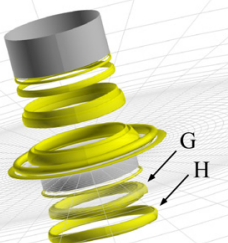

(e) $t=8 T_{f} / 6$

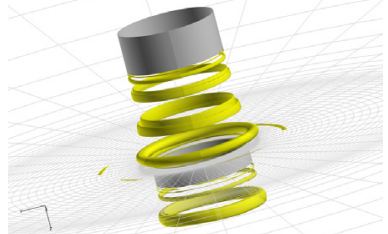

(c) $t=4 T_{f} / 6$

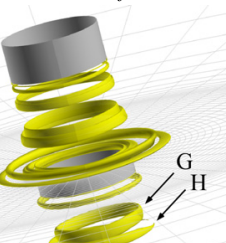

(f) $t=10 T_{f} / 6$

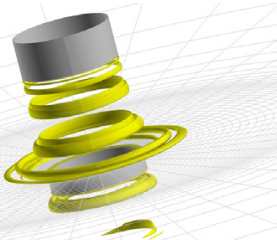

(g) $t=12 T_{f} / 6$

Fig. 12 Visualization of vortical structure by iso-surfaces of the second invariance of the velocity gradient tensor $Q^{*}=29.0\left(1 / T_{f} \sim 292 \mathrm{~Hz}\right)$

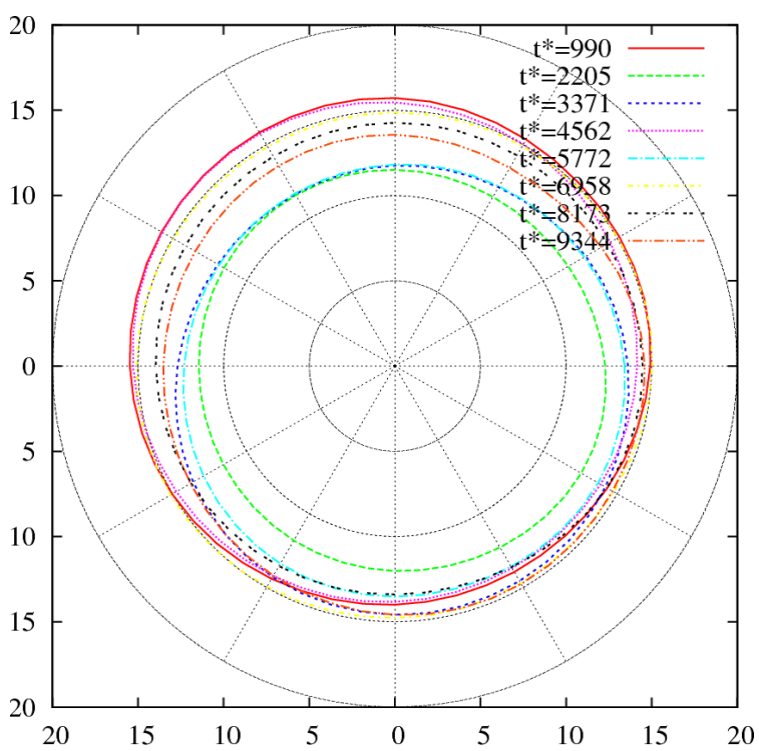

Fig. 13 Polar plots of $\Delta p$ at $(r, z)=\left(d_{0} / 2, L_{i m} / 2\right)$ for times when the pressure fluctuation becomes a local maximum 


\section{Conclusions}

Direct computations and experiments of a hole-tone feedback system were conducted. The mean velocities of an air-jet were 8 and $10 \mathrm{~m} / \mathrm{s}$ in the computations, and $6-13 \mathrm{~m} / \mathrm{s}$ in the experiments. The diameters of the nozzle and the end plate hole used were both $50 \mathrm{~mm}$, and the impingement length between the nozzle and the end plate was $50 \mathrm{~mm}$. After validating the present numerical method against experimental data in terms of qualitative vortical structures and the relationship between the most dominant hole-tone peak frequency and the jet speed, the shear-layer impingement on the hole edge, the resultant propagation of pressure waves and the associated vortical structures are discussed based on the computational results of the air-jet speed of $10 \mathrm{~m} / \mathrm{s}$. As far as the authors know this is the first direct computation of a hole-tone feedback system that successfully predicts its dominant frequency.

(1) When the jet speed was increased in the experiment, initially laminar shear layers became intermittently turbulent and a mode jump in the most dominant hole-tone peak frequency took place at around $u_{0}=12 \mathrm{~m} / \mathrm{s}$. Histerisis was also observed between the cases where $u_{0}$ was increased and where $u_{0}$ was decreased.

( 2 ) High and low pressure regions appeared alternately over a wide area upstream and downstream of the end plate hole. Dominant pressure fluctuations propagated inside the jet, and the frequencies of the pressure fluctuations coincided with those of successive vortices in the jet shear layer.

( 3 ) Non-axisymmetric vortical structures were observed both in the computations and experiments.

\section{Acknowledgements}

This research was supported by the Global COE Program "World Center for Education and Research for Trans-disciplinary Flow Dynamics," of Tohoku University. Computational Resources were provided by Advanced Fluid Information Research Center of Institute of Fluid Science, Tohoku University. The experiment was conducted with cooperation of Mr. Atsushi Totsuka, a technician at Institute of Fluid Science, Tohoku University.

\section{References}

( 1 ) Sondhauss, C., Ueber die beim Ausstromen der Luft enstehenden Tone, Annalen der Physik, Vol. 167, No. 1 (1854), pp. 126-147.

( 2 ) Rayleigh, Lord., Theory of Sound, 2 (1945), pp. 410-412, Dover Publications.

( 3 ) Rossiter, J. E., The Effect of Cavities on the Buffeting of Aircraft, Royal Aircraft Establishment Technical Memorandum, No. 754 (1962).

( 4 ) Chanaud, R. C. and Powell, A., Some Experiments Concerning the Hole and Ring Tone, Journal of the Acoustical Society of America, Vol. 37, No. 5 (1965), pp. 902-911.

( 5 ) Rockwell, D. and Naudascher, E., Self-Sustained Oscillations of Impinging Free Shear Layers, Annual Review of Fluid Mechanics, Vol. 11 (1979), pp. 67-94.

( 6 ) Howe, M. S., Edge, Cavity and Aperture Tones at Very Low Mach Numbers, Journal of Fluid Mechanics, Vol. 330 (1997), pp. 61-84.

( 7 ) Umeda, Y. and Ishii, R., Frequency Characteristics of Discrete Tones Generated in a High Subsonic Jet, AIAA Journal, Vol. 24 (1986), pp. 693-695.

( 8 ) Langthjem, M. A. and Nakano, M., A Numerical Simulation of the Hole-Tone Feedback Cycle Based on an Axisymmetric Discrete Vortex Method and Curle's Equation, Journal of Sound and Vibration, Vol. 288, No. 1-2 (2005), pp. 133-176.

( 9 ) Matsuura, K. and Nakano, M., Direct Computation for the Sound Control of a Hole-Tone Feedback System, Proceedings of the Sixth International Conference Flow Dynamics (2009), pp. 162-163.

(10) Matsuura, K. and Nakano, M., Direct Numerical Simulation of a Hole-Tone Feedback System, CD-ROM Proceedings of International Conference on Jets, Wakes and Separated Flows, (2010), pp. 1-6. 
(11) Howe, M. S., Acoustics of Fluid-Structure Interactions, (1997), Cambridge University Press.

(12) Lele, S. K., Compact Finite Difference Schemes with Spectral-Like Resolution, Journal of Computational Physics, Vol. 103, No. 1 (1992), pp. 16-42.

(13) Gaitonde, D. V. and Visbal, M. R., Padé-Type Higher-Order Boundary Filters for the Navier-Stokes Equations, AIAA Journal, Vol. 38, No. 11 (2000), pp. 2103-2112.

(14) Matsuura, K. and Kato, C., Large-Eddy Simulation of Compressible Transitional Flows in a Low-Pressure Turbine Cascade, AIAA Journal, Vol. 45, No. 2 (2007), pp. 442-457.

(15) Kim, J. W. and Lee, D. J., Generalized Characteristic Boundary Conditions for Computational Aeroacoustics, AIAA Journal, Vol. 38, No. 11 (2000), pp. 2040-2049. 